\title{
Plasmonic silver nanoparticles with atomically precise composition
}

Volume 4 Issue 2 - 2016

\author{
Yangwei Liu \\ Department of Chemistry, Georgetown University, USA
}

Correspondence:Yangwei Liu, Department of Chemistry, Georgetown University, 37 \& O Sts NW, 240 Reiss Bldg, Washington, DC 20057, USA Email y1378@georgetown.edu

Received: September 28, 2016 | Published: October 5, 2016 nanoclusters have generated great research interests for years due to their size-dependent properties. ${ }^{1}$ and wide applications, such as in the field of medicine, ${ }^{2,3}$ biology, ${ }^{4,5}$ sensing, ${ }^{6}$ catalysis, ${ }^{7}$ energy storage, 8 and so on. Large amounts of synthetic and mechanistic studies for a broad range of applications have been reported particularly since the discovery of Au25, which is one of the most stable clusters for gold. ${ }^{9}$ Synthesis of Ag clusters, however, proved to be relatively more challenging mainly due to the poor stability and controllability of silver, as well as possibly different type of interactions between metal and ligand.

Recently, a collaborative study. ${ }^{10}$ led by Professors Nanfeng Zheng, Hannu Hakkinen, and Alison Edwards identified a type of Ag particles that have unique optical properties. In this work, Ag136 and Ag374 species protected by 4-tert-butylbenzenethiolate were chemically synthesized and structurally resolved by X-ray crystallography. It is noteworthy that, although these silver nanoparticles (Ag NPs) were identified with atomically precise composition, these species showed plasmonic optical characteristics. ${ }^{10}$ Atomically precise silver species, taking Ag44 as an example, typically possess characteristic optical absorption peaks and thus were initially described as intensely and broadly absorbing nanoparticles (IBANs). ${ }^{11}$ before structurally resolved. ${ }^{12}$ The ultraviolet-visible absorption (UV-vis) of Ag136 and Ag374 reported in this work was nowhere close to molecular species and showed clear metallic features.

These Ag NPs were observed to have diameters around 2-3 nm which did not come from the irradiation of electron beam as the case of many $\mathrm{Ag}$ or Au nanoclusters. It is known that electron microscopy images are not representative for cluster size characterization due to metal growth and agglomeration under electron beam. However, the high resolution electron microscope images showed that the lattices of these particles were nicely ordered into fivefold twinning, ${ }^{10}$ which was indicative for nanoparticles of face-centered cubic (fcc) structures. ${ }^{13}$

On the other hand, these particles were distinctive from conventional nanoparticles in the sense that they only contained one size rather than mixture of a broad range of particles. The uniformity of the particle size and composition made them unique and interesting to further investigate.

This study opened a door to a more vaguely defined area between nanoparticles and nanoclusters. It showed that atomically precise silver could be prepared with larger size that turned their properties from molecular into metallic type. It could lead to new insights that are helpful for mechanistic investigations and better understanding of the particle formation process.

\section{Acknowledgments}

None.

\section{Conflicts of interest}

None.

\section{References}

1. Kelly K L, Coronado E, Zhao LL et al. The Optical Properties of Metal Nanoparticles: The Influence of Size, Shape, and Dielectric Environment. The Journal of Physical Chemistry B. 2003;107(3):668-677.

2. Sperling RA, Rivera Gil P, Zhang F et al. Biological Applications of Gold Nanoparticles. Chemical Society Reviews. 2008;37(9):1896-1908.

3. De M, Ghosh PS, Rotello VM Applications of Nanoparticles in Biology. Advanced Materials. 2008;20(22):4225-4241.

4. Devi LB, Das SK, Mandal AB Impact of Surface Functionalization of Agnps on Binding and Conformational Change of Hemoglobin $\mathrm{Hb}$ and Hemolytic Behavior. The Journal of Physical Chemistry C. 2014;118(51):29739-29749.

5. Loza K, Diendorf J, Sengstock C et al. The Dissolution and Biological Effects of Silver Nanoparticles in Biological Media. Journal of Materials Chemistry B. 2014;2:1634-1643.

6. Mayer KM, Hafner JH Localized Surface Plasmon Resonance Sensors. Chemical Reviews. 2011;111(6):3828-3857.

7. Astruc D, Lu F, Aranzaes JR Nanoparticles as recyclable catalysts: the frontier between homogeneous and heterogeneous catalysis. Ange Chemie Inter Ed. 2005;44(48):7852-7872.

8. Frey NA, Peng S, Cheng K et al. Magnetic nanoparticles: synthesis, functionalization, and applications in bioimaging and magnetic energy storage. Chemical society reviews. 2009;38(9):2532-2542.

9. Jin R, Zeng $\mathrm{C}$, Zhou $\mathrm{M}$ et al. Atomically precise colloidal metal nanoclusters and nanoparticles: fundamentals and opportunities. Chemical reviews. 2016;116 (18):10346-10413.

10. Yang H, Yu Wang, Xi Chen et al. Plasmonic Twinned Silver Nanoparticles with Molecular Precision. Nat Commun 7. 2016

11. Bakr OM, Amendola V, Aikens CM et al. Silver nanoparticles with broad multiband linear optical absorption. Angewandte Chemie International Ed. 2009;48(32):5921-5926. 
12. Harkness KM, Yun Tang, Amala Dass et al. Ag44(SR)304-: a silverthiolate superatom complex. Nanoscale. 2012;4(14):4269-4274.
13. Xia Y, Xiong Y, Lim B et al. Shape-controlled synthesis of metal nanocrystals: simple chemistry meets complex physics? Angewandte Chemie International Edition. 2009;48(1):60-103. 